\title{
On the structure of contact binaries
}

\author{
H. Kähler ${ }^{\star}$ \\ Hamburger Sternwarte, Gojenbergsweg 112, 21029 Hamburg, Germany \\ Received 3 April 2002 / Accepted 13 September 2002

\begin{abstract}
In a preceding paper a simple model for contact binaries has been proposed, based on the usual assumption that the energy sources/sinks caused by the interaction of the components occur only in the secondary's/primary's outer layers. Here the model is applied to a typical late-type system.

First we imposed the restriction that the fractional extent in mass of the sources/sinks in the layers above the critical surface is the same in both components, and found solutions evolving in thermal cycles. If the energy transfer is assumed to be sufficiently effective, loss of contact is avoided. Otherwise the cycle consists of a long quiet phase in good contact and a short violent phase with rapid changes between contact, semidetached, and detached configurations. In both cases there are cycles in which the temperatures of the components are similar in the largest part of the time. Other observational tests are also passed.

Next we removed the restriction. In the resulting model with three free parameters we found (besides cycles) a large range of solutions in stable thermal equilibrium which are compatible with the observations.

From a theoretical viewpoint however all these solutions are unsatisfactory since suffering from several inconsistencies. This result shows that the usual assumption on the energy sources and sinks is too restrictive. We are led to look for modifications of the model which remove the inconsistencies. This will be done in a forthcoming paper.
\end{abstract}

Key words. stars: binaries: close

\section{Introduction}

In a preceding paper (Kähler 2002, hereafter K1) the structure equations of contact binaries have been discussed. Serious uncertainties have been shown to concern only the energy transfer by circulation currents from the primary to the secondary. When studying thermal cycles of contact systems (and possibly also in more general cases) these uncertainties can be expressed as (so far unknown) functions. A simple tentative choice of these functions has been made, and an evolution code for contact systems has been presented.

Here the code will be used to investigate unevolved systems with the physical parameters

$M=1.6 M_{\odot}, \quad J_{52}=0.4, \quad X=0.7, \quad Z=0.02$,

where $J_{52}$ denotes the angular momentum in $10^{52}$ cgs-units. With a mass ratio of about 0.4 and a period of $\sim 0.3 \mathrm{~d}$ they represent typical late-type systems. We shall adhere to the notation in K1. The parameters listed in Eq. (47) of K1 will be kept fixed, and the extent of turbulent layers will be assumed to be determined by the Schwarzschild criterion $\left(i_{\mathrm{t}}=0\right)$. Free parameters are $f_{\mathrm{E}}$ and $\alpha_{i}(i=1,2)$. While $f_{\mathrm{E}}$ is a measure for the efficiency of the energy transfer, $\alpha_{i}$ denotes the extent of the layers with energy sources or sinks caused by the circulation currents in the envelope of the component $i$, expressed as fractional mass of the layers above the critical surface.

* e-mail: hkaehler@hs.uni-hamburg.de
Table 1. Parameters of systems (labeled by the number in the first column) and global properties of the cycles (see text).

\begin{tabular}{llllllll}
\hline \hline & $\alpha$ & $f_{\mathrm{E}}$ & $\tau$ & $\Delta T_{\mathrm{e}}$ & $\Lambda_{\max }$ & $F_{\max }$ & $F_{2, \min }$ \\
\hline 1 & 0.02 & 0.5 & 0.462 & -434 & 0.439 & 0.0180 & 0.0021 \\
2 & 0.015 & 0.5 & 0.327 & -256 & 0.410 & 0.0176 & 0.0115 \\
3 & 0.015 & 0.4 & 0.389 & -322 & 0.421 & 0.0187 & 0.0086 \\
4 & 0.015 & 0.35 & 0.458 & -440 & 0.441 & 0.0196 & 0.0040 \\
5 & 0.015 & 0.3 & 0.508 & -467 & 0.449 & 0.0204 & -0.035 \\
6 & 0.015 & 0.2 & 0.547 & -456 & 0.441 & 0.0223 & -0.07 \\
7 & 0.015 & 0.1 & 0.567 & -317 & 0.413 & 0.0258 & -0.08 \\
8 & 0.015 & 0.05 & 0.580 & -175 & 0.386 & 0.0295 & -0.09 \\
\hline
\end{tabular}

\section{Thermal cycles}

To reduce the number of free parameters first we assumed that $\alpha_{1}=\alpha_{2}$. Dropping the index for the component we obtained a model with only two free parameters $f_{\mathrm{E}}, \alpha$.

We found systems evolving in thermal cycles. One of the cycles was obtained starting from an artificial initial configuration as described in $\mathrm{K} 1$ and following the evolution for a sufficiently long time. The other cycles were obtained from the first one performing continuous changes of the parameters. We investigated representative systems defined by the first three columns in Table 1. 


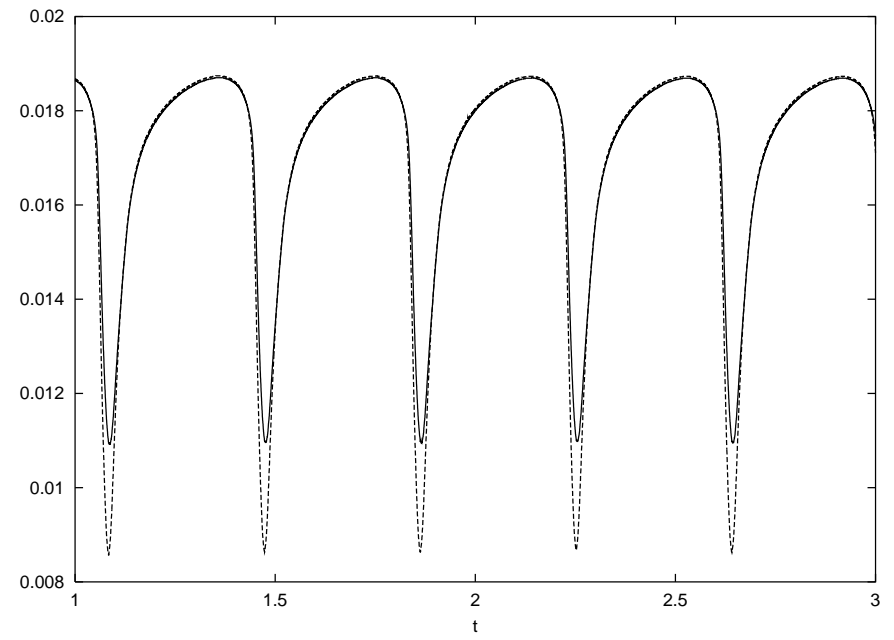

Fig. 1. The degree of contact of the primary (solid line) and the secondary (dashed line) in system 3.

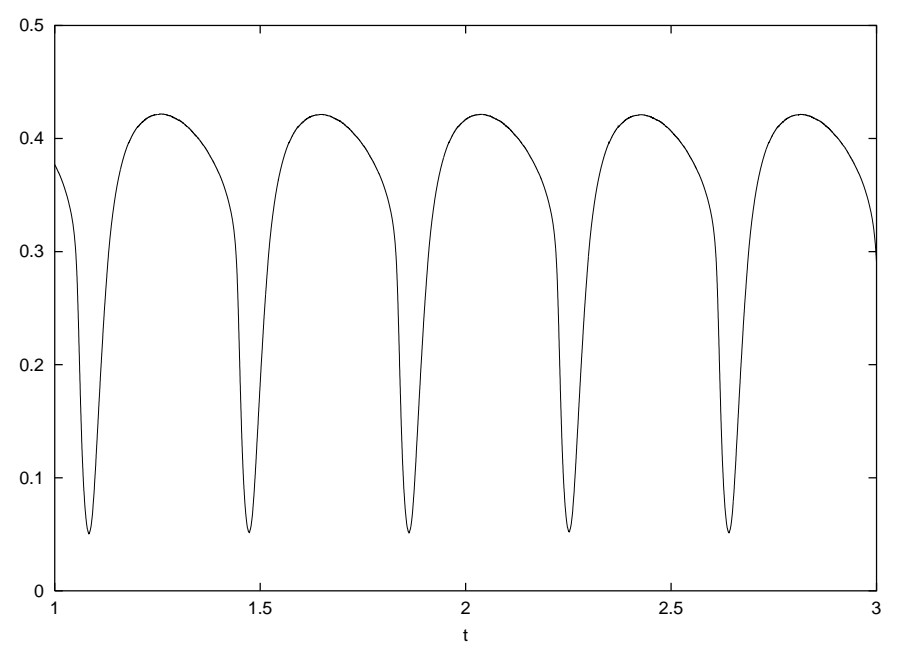

Fig. 2. The energy $\Lambda$ (in solar units) transferred by circulation currents in system 3 .

\subsection{Cycles without loss of contact}

If the energy tranfer is very effective (i.e. $f_{\mathrm{E}}$ sufficiently large) and the common turbulent envelope very shallow (i.e. $\alpha$ sufficiently small), the system evolves in thermal cycles without loss of contact. This is the case in systems 1-4. The cycles for these systems are similar. As an example with good light curves we shall consider the cycle of system 3. Figures 1-6 show the changes of characteristic quantities during the cycle. (Unless stated otherwise, throughout this paper the time will be measured in units of $10^{7}$ years.) The maximum and the minimum of the period can be used to divide the cycle into a long phase (lasting about 80 percent of the time) and a short phase. In the long phase the Roche equipotential condition is satisfied in a close approximation, matter is flowing from the secondary to the primary, the period and the radii of the components are increasing, and the system is in good thermal contact with a temperature difference between the components not larger than about $300 \mathrm{~K}$. Conversely, in the short phase the Roche equipotential condition is violated, matter is flowing from the primary

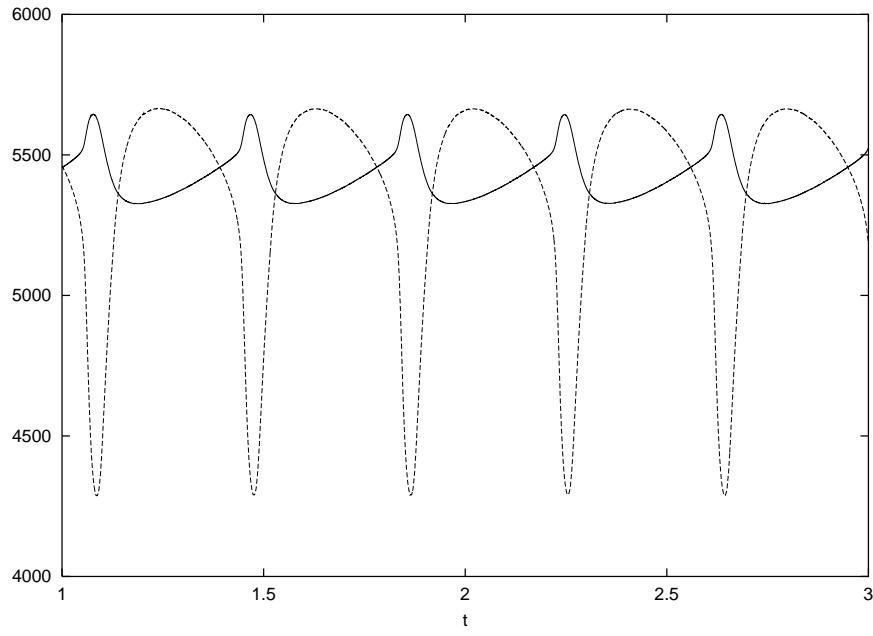

Fig. 3. The effective temperature of the primary (solid line) and the secondary (dashed line) in system 3 .

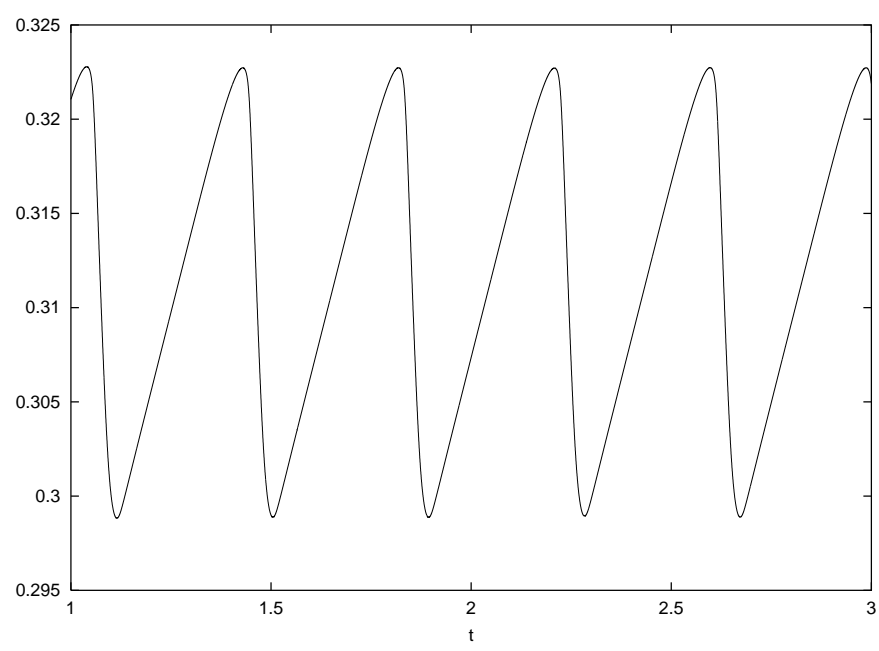

Fig. 4. The period (in days) in system 3 .

to the secondary at a much larger rate than in the long phase, period and radii are decreasing, and the system is in very shallow contact and thus also in poor thermal contact, with a large temperature difference between the components. In both phases the accretion luminosity is unimportant.

Included in Table 1 are some characterisic properties of the cycles, i.e. $\tau$ (the cycle length), $\Delta T_{\mathrm{e}}$ (the temperature difference $T_{\mathrm{e} 1}-T_{\mathrm{e} 2}$ when $T_{\mathrm{e} 2}$ is maximum), $\Lambda_{\max }$ (the maximum of the energy tranfer $\Lambda$ in solar units), $F_{\max }$ (the maximum degree of contact), and $F_{2 \text {,min }}$ (the minimum of the secondary's degree of contact). A comparison of these properties in systems 1-4 shows how the cycle depends on the parameters $f_{\mathrm{E}}$ and $\alpha$. The main effect of an decrease in $f_{\mathrm{E}}$ is an increase of the amplitudes of the variations during the cycle. The increase concerns all quantities of the system, including the cycle length. In system 4 the parameter $f_{\mathrm{E}}$ is so small that loss of contact is just avoided. An increase in $\alpha$ has a similar effect, as it is seen comparing systems 1 and 2 .

As mentioned above, we have assumed that the extent of turbulent layers is determined by the Schwarzschild criterion, i.e. that $i_{\mathrm{t}}=0$. To see the effect of an enhanced influence of 


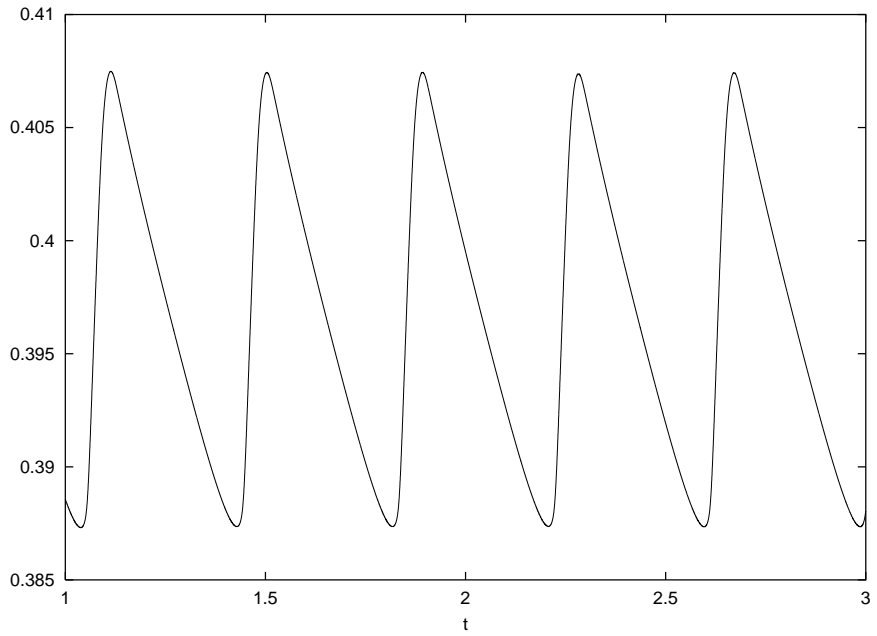

Fig. 5. The mass ratio in system 3 .

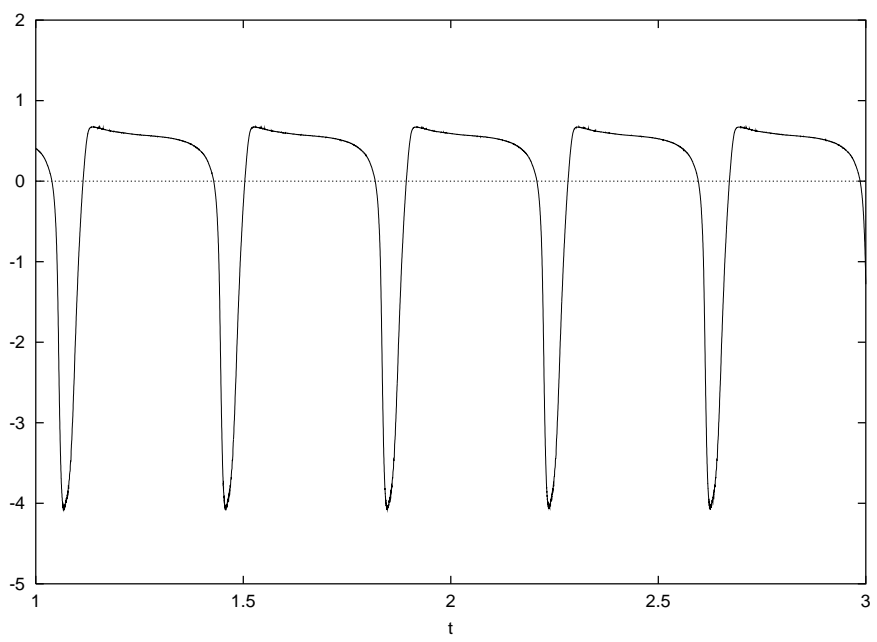

Fig. 6. The rate of mass exchange $\dot{M}_{1}$ (in $10^{-8}$ solar masses per year) in system 3.

turbulence, we calculated also the cycle of system 3 assuming $i_{\mathrm{t}}=1$. The effect was mainly an increase in the cycle length ( $\tau=0.406)$. Other effects turned out to be very small.

\subsection{Cycles with loss of contact}

If loss of contact occurs, the maximum and the minimum of the period can again be used to divide the cycle into two phases. In a long quiet phase the system is in good contact. A short violent phase contains rapid changes between contact, semidetached, and detached configurations. These rapid changes are accompanied by sharp peaks in the rates of mass and energy exchange, the luminosities, and other quantities.

This is the case in systems $5-8$. For these systems the quantities $\Delta T_{\mathrm{e}}, \Lambda_{\max }, F_{\max }$ in Table 1 refer to the long phase. In this phase the systems behave similarly as in the long phase of cycles without loss of contact. The effect of a decrease in $f_{\mathrm{E}}$ (an increase of the amplitude of all variations during a cycle) is also similar. System 8 appears to be close to a limiting case. For smaller values of $f_{\mathrm{E}}$ we encountered severe numerical difficulties when trying to calculate cycles.

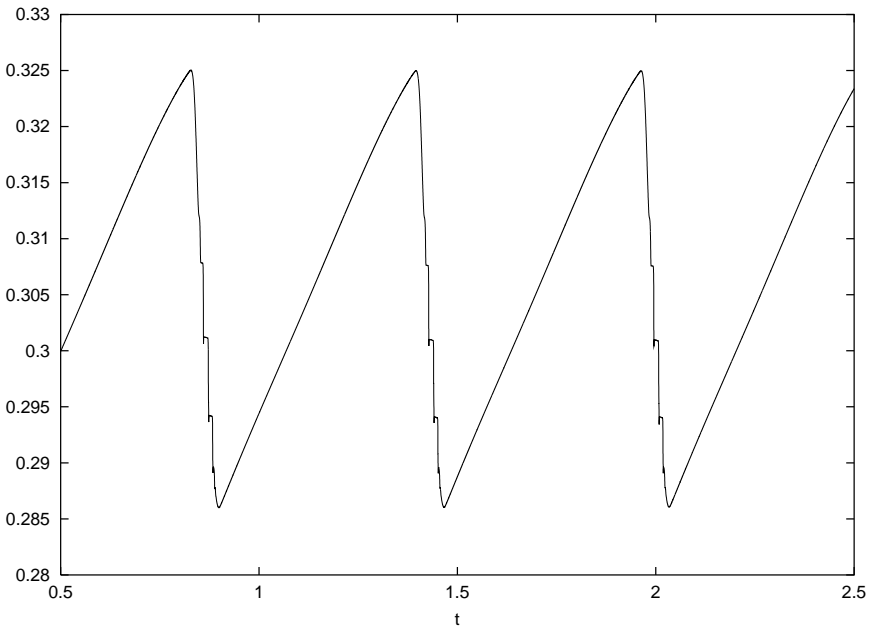

Fig. 7. The period (in days) in system 7.

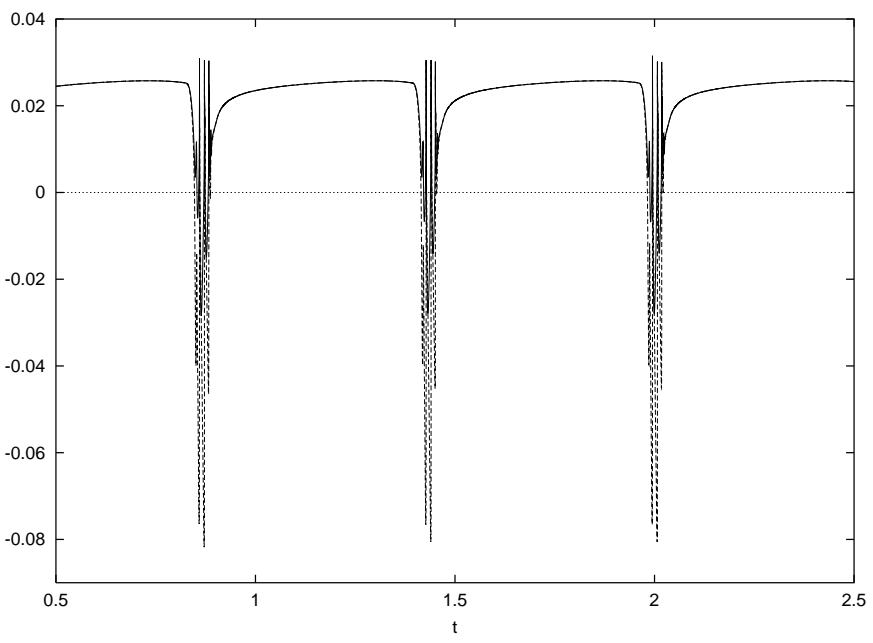

Fig. 8. The degree of contact of the primary (solid line) and the secondary (dashed line) in system 7.

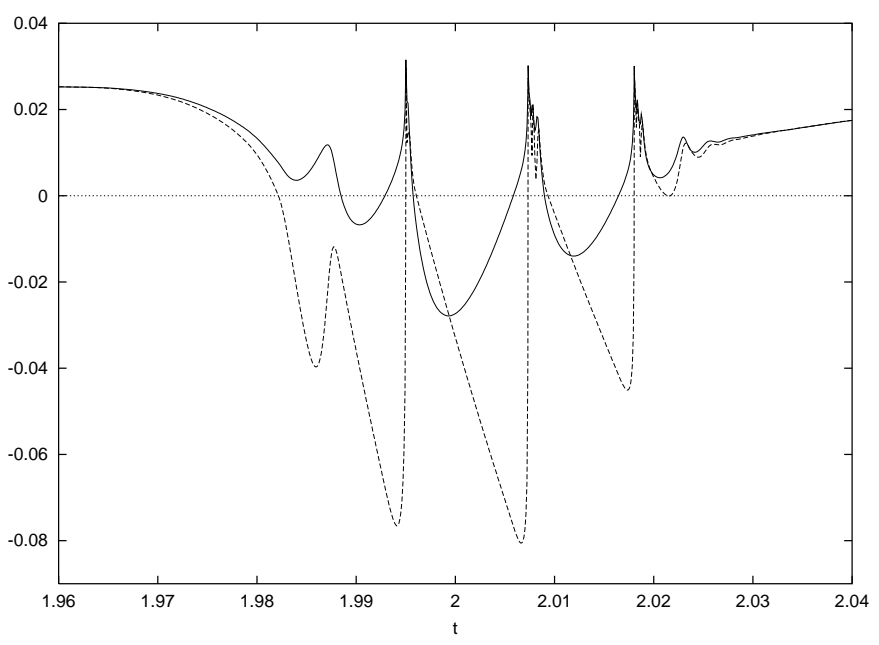

Fig. 9. The degree of contact of the primary (solid line) and the secondary (dashed line) in a violent phase of system 7 .

As an example with good light curves consider the cycle of system 7. The run of the period (Fig. 7) defines the two phases and shows that the violent phase occupies only a small fraction 


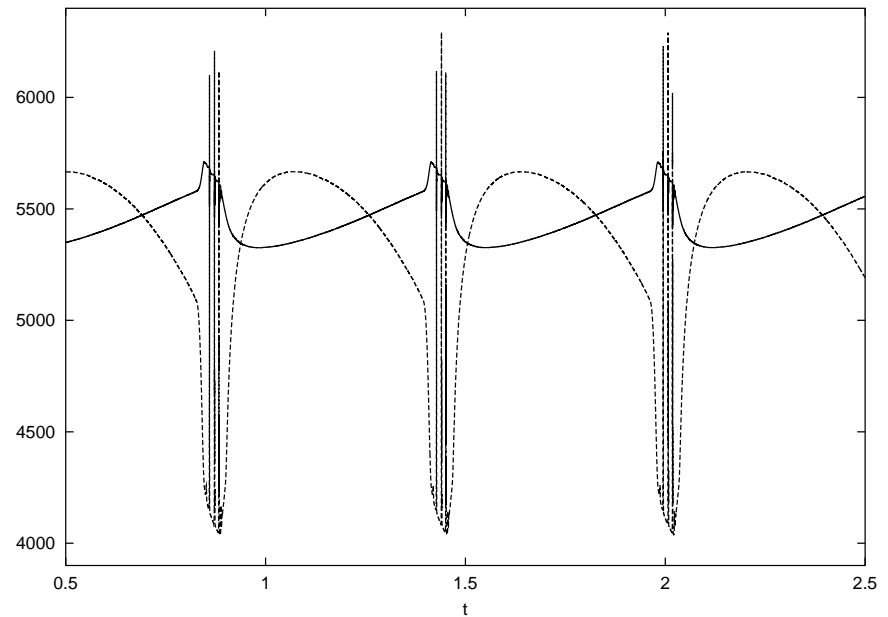

Fig. 10. The effective temperature of the primary (solid line) and the secondary (dashed line) in system 7.

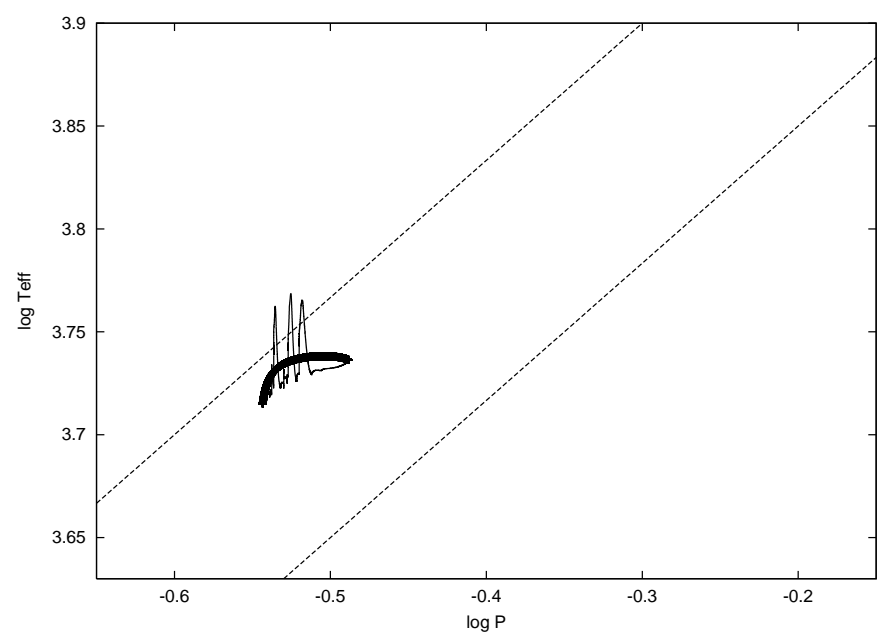

Fig. 11. The cycle of system 7 in the period-colour diagram (see text).

of the cycle. This is confirmed in Fig. 8. Details of the violent phase are shown in Fig. 9. Note that all details are well-defined and that the behaviour of the system is not chaotic but strictly periodic.

During the peaks in the violent phase the mass flow increases (for a very short time) to enormous values of $\dot{M}_{1} \sim$ $-5 \times 10^{-6}$ solar masses per year, and this is accompanied by peaks in the accretion luminosity of $L_{\text {acc }} \sim 0.5 L_{\odot}$. In the neighbourhood of the peaks the computations are difficult since extremely small time steps are required. The difficulties arise in the secondary where the core luminosity is very small and the adjustment of the temperature gradient causes problems.

Most important from an observational viewpoint are the temperatures of the components. Figure 10 shows that the temperature difference is smaller than about $300 \mathrm{~K}$ in about 80 percent of the time. This is as in system 3 .

Considering all systems (without and with loss of contact during the cycle) together, and disregarding details in the short phase, the global properties of the cycles turn out to depend continuously on the parameters. For some properties this can

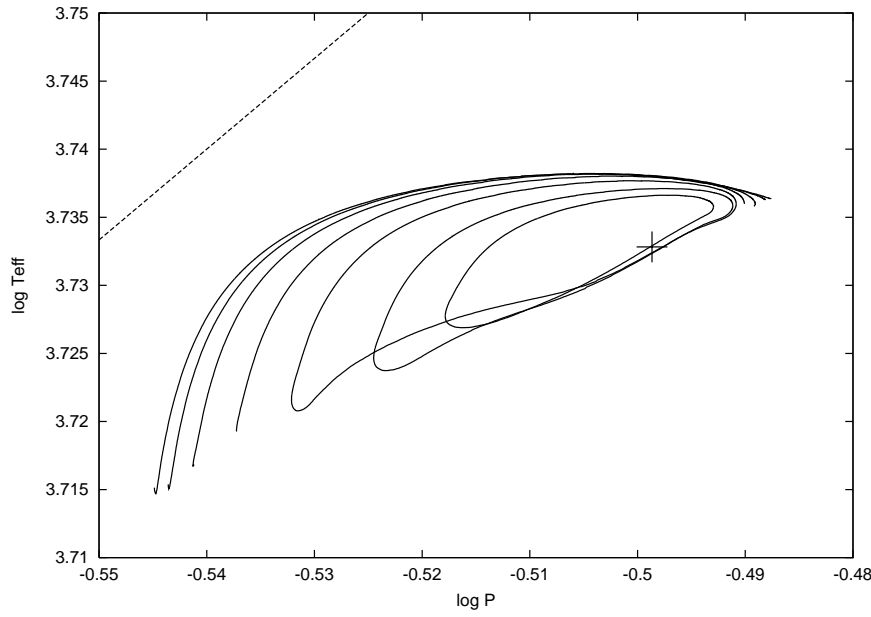

Fig. 12. The cycles of systems $1-8$ in the period-colour diagram. The cross represents the (unstable) equilibrium configuration with the parameters of system 3 (see text).

be seen in Table 1, and for other properties it will become clear in the following subsection.

\subsection{The cycles in the period-colour diagram}

The results obtained so far are compatible with two basic observational facts, the light curves and the preference for shallow contact. A third basic observational fact is the period-colour relation. As shown by Eggen $(1961,1961)$, the observed systems are in this diagram found within the strip

$1.5 \log T_{\text {eff }}-\log P=5.975 \ldots 6.15$

(where $P$ denotes the period in days) which is limited by the dashed lines in Fig. 11 (cf. Kähler \& Fehlberg 1991). Also shown is the cycle of system 7 . The long phase (marked by a thick line) is located within the strip, as well as the short phase apart from three peaks. Accordingly, system 7 is compatible with the period-colour relation.

Figure 12 shows the cycles of systems $1-8$ in an enlarged part of the period-colour diagram. To be more precise, shown are the full cycles of sytems $2-4$ and the long phase of systems $5-8$. (The cycle for system 1 almost coincides with the cycle of system 2.) The smallest cycle belongs to system 2 with the largest value of $f_{\mathrm{E}}$. Smaller values of $f_{\mathrm{E}}$ give larger cycles, in accordance with the general result that the amplitudes of the variations increase when $f_{\mathrm{E}}$ decreases. All cycles are compatible with the period-colour relation. Figure 12 shows also that the averaged location of a system during a cycle in the periodcolour diagram depends only weakly on the parameters $f_{\mathrm{E}}, \alpha$.

\subsection{The structure of the components}

As an example for the structure of a system in good contact consider system 3 when the secondary's temperature is maximum. Figure 13 shows the run of entropy (per nucleus divided by Boltzmann's constant) in both components as a function of the relative radius. The flat parts for $r / R \gtrsim 0.7$ 


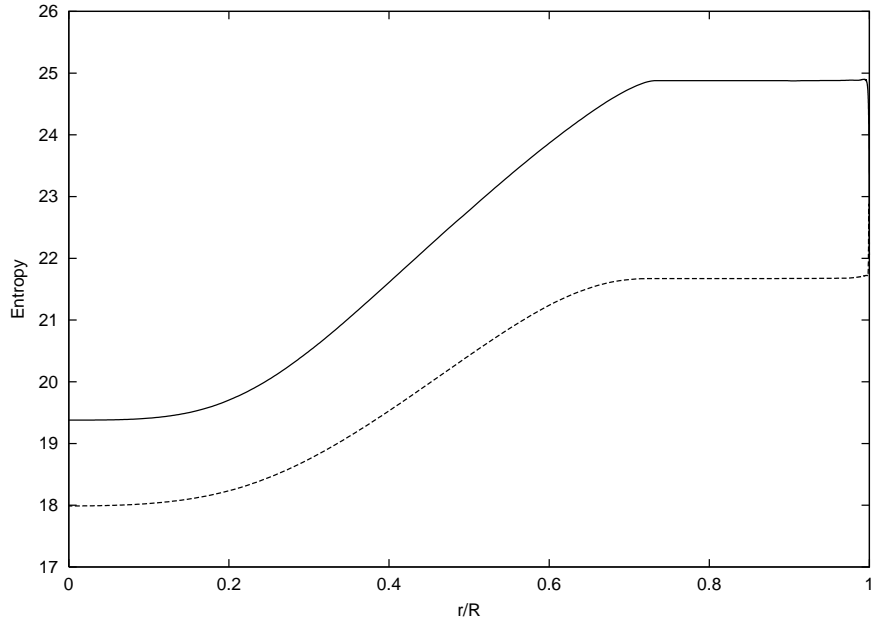

Fig. 13. The run of entropy in the primary (solid line) and the secondary (dashed line). For details near the surface see Fig. 14.

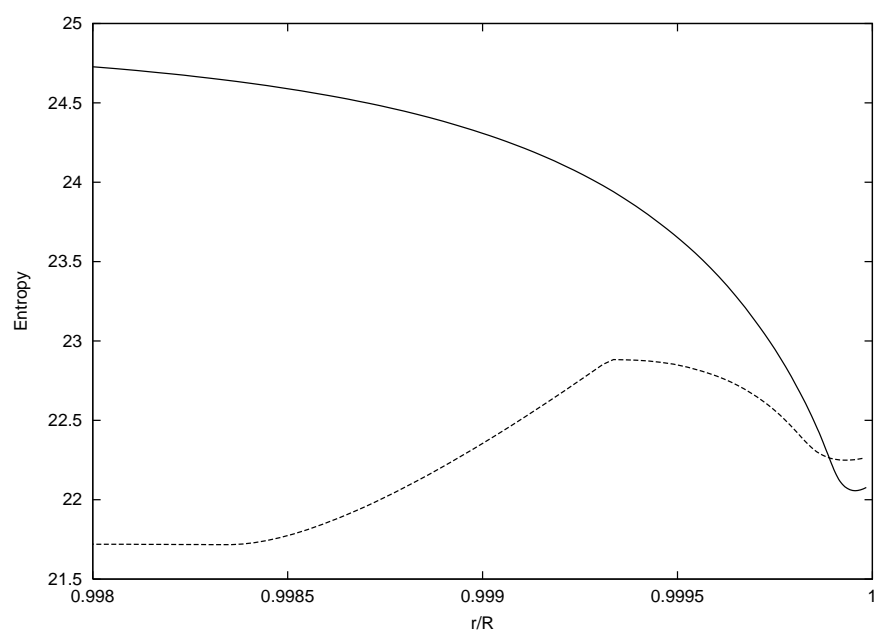

Fig. 14. The run of entropy in the outermost layers of the primary (solid line) and the secondary (dashed line).

indicate large convective regions with very different specific entropies. Details in the outermost layers (above the critical surface) are shown in Fig. 14. The secondary's layers with $0.9984<r / R<0.9994$ are radiative, as indicated by the increase of entropy. Above these layers there is again convection as the result of the energy supply from the primary.

In the secondary's outer layers below the energy sources the luminosity is extremely low. Figure 15 shows that in these layers the core luminosity has almost completely been exhausted by negative values of the $\varepsilon_{\mathrm{g}}$-terms in the energy balance, i.e. by the expansion of the secondary.

As indicated by the small value of $\alpha$ and as illustrated in these diagrams, the energy transfer between the components occurs in the outermost layers (in the secondary in the layers with $\log \rho \leq-6.16)$, i.e. in the extreme superadiabatic layers. This feature and the large difference of the entropies in the adiabatic parts of the large convective zones are similar as in models in thermal equilibrium discussed by Moss \& Whelan (1973). These models have been criticized by Hazlehurst (1974) for two reasons: (1) The configurations are considered to be

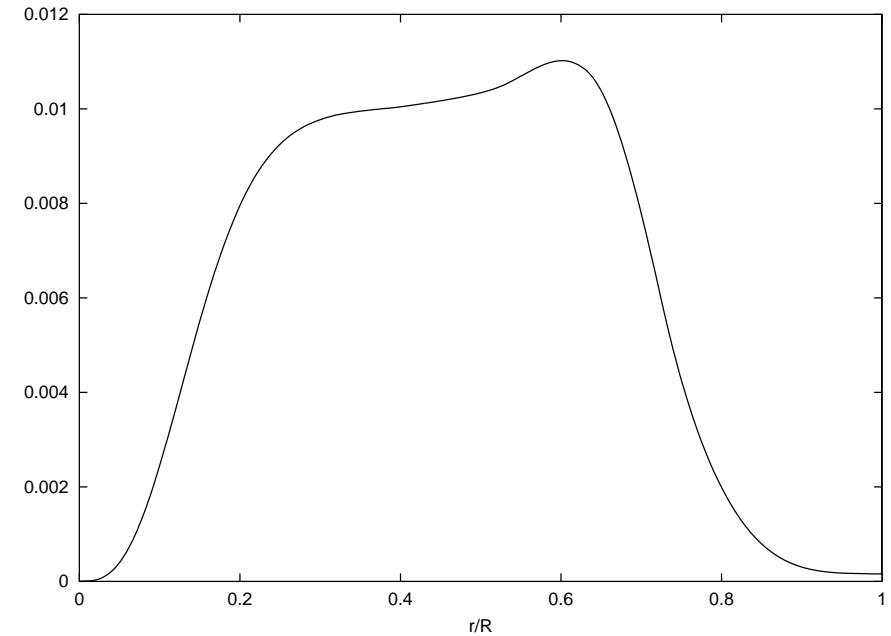

Fig. 15. The run of luminosity (in solar units) in the secondary. The peak near the surface escapes notice.

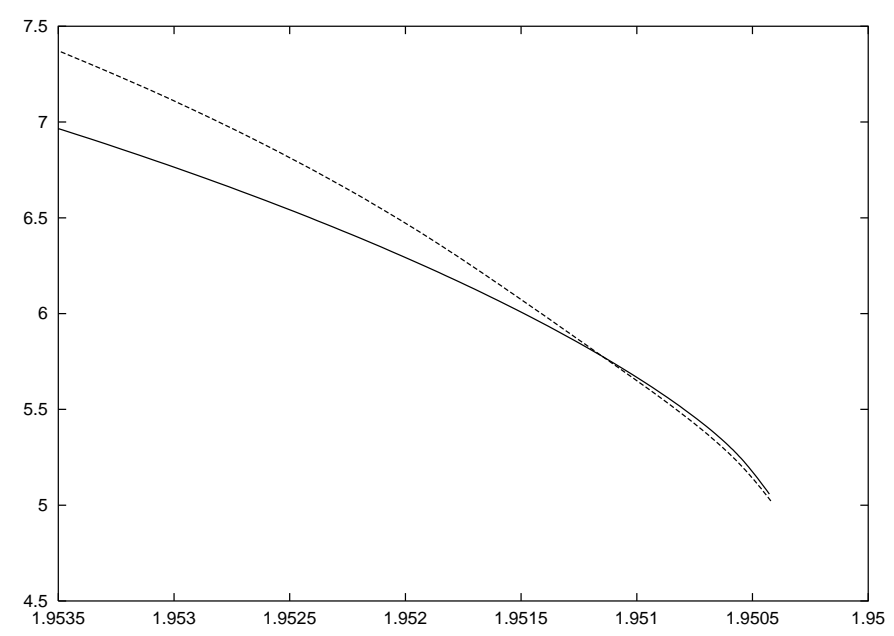

Fig. 16. The run of the pressure in the layers avove the critical surface. Shown is $\log P_{i}$ in dependence on the normalised effective potential $\varphi_{i}$ for $i=1$ (solid line) and $i=2$ (dashed line).

thermally unstable, and (2) an energy transfer in the extreme superadiabatic layers is impossible since the heat capacity of these layers is too small. As far as the present results are concerned, the first reason is unimportant since the stability arguments have been withdrawn by Hazlehurst \& Refsdal (1980) and since the present configurations are anyway in thermal imbalance. The second reason however is important and shows that the cycles obtained are inconsistent from a theoretical viewpoint.

A second inconsistency concerns again the luminosity transfer. According to Eq. (48) in K1 the transfer is calculated from physical properties at the critical surface, i.e. at the very base of the common envelope. These properties are characteristic for the bulk of the neck (since pressure and density in the neck are largest at the L1 point), but not for the outermost part where the transfer is assumed to occur if $\alpha$ is very small. This shows again that solutions for very small values of $\alpha$ are inconsistent. 


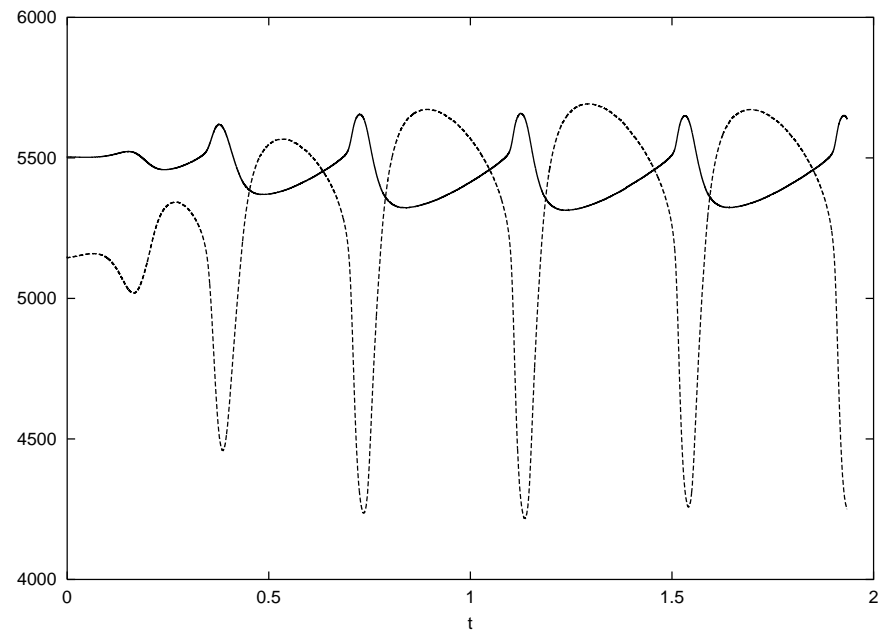

Fig. 17. Evolution from an unstable equilibrium configuration into the cycle of system 3 . Shown are the effective temperature of the primary (solid line) and of the secondary (dashed line).

A third inconsistency concerns the distribution of pressure in the outer layers. In the configuration under discussion the rate of mass exchange is very low $\left(\dot{M}_{1} \simeq 6 \times 10^{-9}\right.$ solar masses per year). Figure 16 shows the run of pressure in the common envelope, i.e. in the layers above the critical surface. Plotted is $\log P_{i}$ as a function of the normalised effective potential $\varphi_{i}(i=1,2)$. In a common envelope in strict hydrostatic equilibrium (absence of internal mass motions) all thermodynamic quantities would be constant on equipotential surfaces on account of Poincaré's theorem, and the two curves in Fig. 16 would coincide. This is approximately the case in the outermost layers, but not in the other layers where the secondary's pressure is much larger than the primary's pressure. Since the luminosity transfer requires internal mass motions, departures from hydrostatic equilibrium are necessary. In a consistent treatment they must be compatible with the low rate of mass exchange. This is not the case. Since pressure differences on equipotential surfaces drive mass motions, according to Fig. 16 mass is flowing mainly form the secondary to the primary in the bulk of the layers above the critical surface, and at a much lower rate from the primary to the secondary in the outermost layers. Accordingly, the pressure distribution is not compatible with the low rate of mass exchange and the solution is inconsistent also from this viewpoint.

\section{Solutions in thermal equilibrium}

Having studied the case of $\alpha_{1}=\alpha_{2}$, next we abandoned this restriction. Starting from a configuration of the cycle for system 3 and increasing continuously the parameter $\alpha_{1}$ we found that for $\alpha_{1}=0.1$ the cycle dies out and the system evolves into a configuration in stable thermal equilibrium. Assuming next thermal equilibrium and changing the parameters we obtained a survey of the equilibrium configurations in dependence on the parameters $f_{\mathrm{E}}, \alpha_{1}, \alpha_{2}$. We are confident that the survey is complete except for configurations with an extremely large temperature difference between the components. All configurations have the charge $c=1$, which is necessary but not sufficient for stability, cf. Kähler et al. (1986).

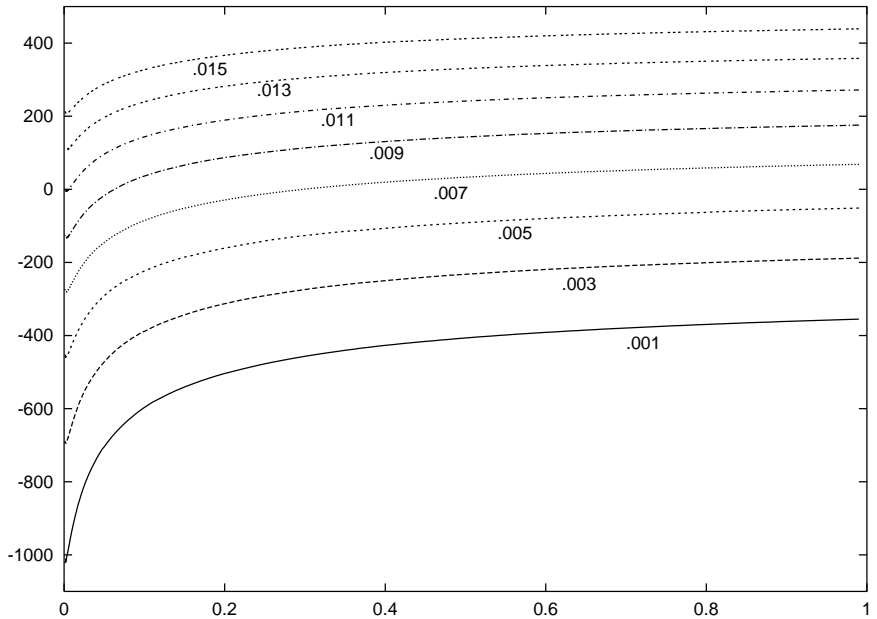

Fig. 18. The temperature difference in equilibrium configurations as a function od $\alpha_{1}$ for $f_{\mathrm{E}}=0.5$ and different values of $\alpha_{2}$.

Some of the configurations are unstable. An example is the equilibrium configuration with the parameters of system 3 . In the period-colour diagram (Fig. 12) this configuration is represented by a cross. Figure 17 illustrates the evolution from this configuration into the cycle of system 3 . Solutions with $\alpha_{1} \gg \alpha_{2}$, however, are usually (perhaps always) stable. This was checked not by an eigenvalue analysis but by evolutionary calculations following an initial perturbation.

The temperature difference between the components in dependence on $\alpha_{1}$ and $\alpha_{2}$ in the case of $f_{\mathrm{E}}=0.5$ is shown in Fig. 18. While the dependence on $\alpha_{1}$ is weak only (except for very small values of $\alpha_{1}$ ), the dependence on $\alpha_{2}$ is strong. This reflects the fact that the secondary's structure is much more influenced by the interaction between the components than the primary's structure. We are interested only in solutions with a small temperature difference. Solutions of this type exist for any value of $\alpha_{1}$, but they require values of $\alpha_{2}$ which are very small compared to unity. In the case of smaller values of $f_{\mathrm{E}}$ this difficulty is even more pronounced. For $f_{\mathrm{E}}=0.1$ this is shown in Fig. 19. Since the entropy distribution is similar as in Figs. 14 and 15, the solutions are of the type proposed by Moss \& Whelan (1973). Other solutions with good light curves do not exist.

We conclude that a small temperature difference requires an energy transfer in the secondary's extreme superadiabatic layers. In configurations with this property the pressure differences on equipotential surfaces in the common envelope are similar as in Fig. 16 and thus in conflict with the vanishing net rate of mass transfer in systems in thermal equilibrium. Accordingly, we encounter the same inconsistencies as in the case of the cycles discussed in the previous section.

\section{Summarizing and concluding remarks}

Applying the model for contact binaries proposed in $\mathrm{K} 1$ to an unevolved system with typical physical parameters we found a variety of solutions which are compatible with the observations, i.e. with the light curves, the period-colour relation, and the preference for shallow contact. These solutions include 


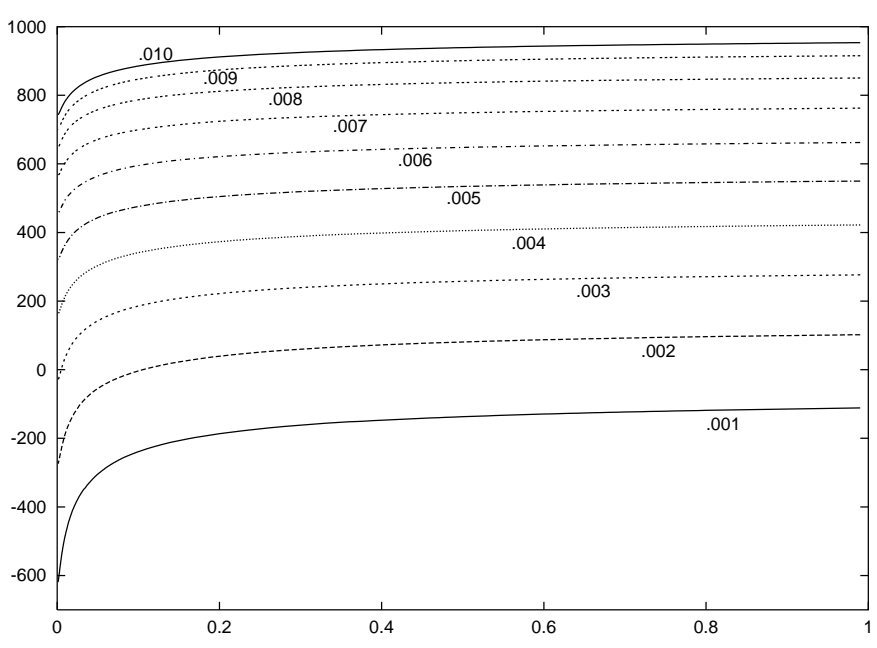

Fig. 19. The temperature difference in equilibrium configurations as a function od $\alpha_{1}$ for $f_{\mathrm{E}}=0.1$ and different values of $\alpha_{2}$.

configurations in stable thermal equilibrium as well as thermal cycles (either without or with loss of contact) in which the temperatures of the components differ not more than about $300 \mathrm{~K}$ in about 80 percent of the time.

From a theoretical viewpoint, however, all these solutions are inconsistent. Good light curves (i.e. similar temperatures of the components) require $\alpha_{2} \ll 1$, which means that the energy transfer in the secondary occurs in the extreme superadiabatic layers. This is impossible as shown by Hazlehurst (1974).

A further inconsistency of solutions with $\alpha_{2} \ll 1$ arises since the luminosity transfer is calculated from physical properties at the critical surface which are not representative for the outermost layers.
A third inconsistency concerns the large difference in pressure and density between the components in the bulk of the layers above the critical surface.

Solutions which are reasonable from an observational as well from a theoretical viewpoint do not exist. Accordingly, the model is too restrictive. This might have been expected since the model is based on the usual assumption (investigated since decades) that the energy sources/sinks caused by the interaction of the components occur only in the secondary's/primary's outer layers. In fact, all solutions compatible with the observations are essentially of the type proposed long ago by Moss \& Whelan (1973).

The model here investigated is a step towards a consistent description of contact binaries. As the discussion in $\mathrm{K} 1$ has shown, there is much more freedom in the structure equations. For this reasons theoretical problems are useful since they offer the opportunity to remove the inconsistencies and to reduce the theoretical uncertainties. In a next step we shall try to solve the problems here encountered with slight modifications of the present model. We refer to a forthcoming publication.

\section{References}

Eggen, O. J. 1961, R. Greenwich Obs. Bull., 31

Eggen, O. J. 1967, Mem. R. Astron. Soc., 70, 111

Hazlehurst, J. 1974, A\&A, 36, 49

Hazlehurst, J., \& Refsdal, S. 1980, A\&A, 84, 200

Kähler, H. 1995, A\&A, 294, 497

Kähler, H. 2002, A\&A, 395, 899, preceding paper (K1)

Kähler, H., \& Fehlberg, H.-J. 1991, A\&A, 252, 137

Kähler, H., Matraka, B., \& Weigert A. 1986, A\&A, 159, 317

Moss, D. L., \& Whelan, J. A. J. 1973, MNRAS, 161, 239 